Article

\title{
Synergistic Effect of Nanodiamond and Phosphate Ester Anti-Wear Additive Blends
}

\author{
Biplav Acharya ${ }^{1, *}\left(\mathbb{D}\right.$, Keshav S. Avva ${ }^{2}$, Binita Thapa ${ }^{1}$, Tyler N. Pardue ${ }^{3}$ and \\ Jacqueline Krim 1,* (i) \\ 1 Department of Physics, North Carolina State University, Raleigh, NC 27695, USA; bthapa@ncsu.edu \\ 2 Department of Mechanical and Aerospace Engineering, North Carolina State University, \\ Raleigh, NC 27695, USA; ksavva@ncsu.edu \\ 3 Department of Materials Science and Engineering, North Carolina State University, Raleigh, NC 27695, USA; \\ tnpardue@ncsu.edu \\ * Correspondence: bachary@ncsu.edu (B.A.); jkrim@ncsu.edu (J.K.)
}

Received: 20 May 2018; Accepted: 13 June 2018; Published: 18 June 2018

check for updates

\begin{abstract}
Nanodiamonds are known to improve tribological performance when added to lubricants, but their impact on additives that may already be present in the lubricant is poorly documented. Here, we report on a study of their effects on thermal reaction films formed from tricresyl phosphate (TCP) on Fe substrates immersed in a dibasic ester basestock when blended with TCP. Thermal reaction film formation temperatures were recorded in-situ by monitoring the reaction film formation on both Fe and air baked Fe surfaces using a quartz crystal microbalance (QCM). The nanodiamonds were found to raise the thermal reaction film formation temperature by $18{ }^{\circ} \mathrm{C}$, possibly by raising the activation energy for the reaction, but they were not observed to affect the thickness or rate of formation of the films. The nanodiamonds, moreover, were observed to trigger thermal reaction film formation on air baked Fe surfaces that otherwise were highly resistance to reaction film formation. The surface morphology, roughness, and thickness of the thermal reaction films, as measured by atomic force microscopy (AFM), are reported as well as their chemical compositions, as studied with Electron Dispersive X-ray Spectroscopy (EDS). The coefficients of friction measured on the thermal reaction films during dry solid-solid contact are also reported.
\end{abstract}

Keywords: phosphate esters; nanodiamonds; TCP; anti-wear; QCM

\section{Introduction}

Organophosphorus compounds are commonly used as anti-wear additives in automotive and aviation lubrication systems [1-3]. Anti-wear additives have vital importance under extreme pressure (EP) conditions where the oil film between contacting surfaces is not sufficiently thick to prevent direct contact between the surface asperities. In such conditions, ideal additives form robust anti-wear films that adhere strongly to the surfaces and also withstand higher temperatures, particularly in aviation engines that operate at higher temperatures and higher speeds [4,5]. Phosphate esters and (metal) thiophosphate esters have been used as lubricant additives for more than 50 years in both the automotive and aviation industries. Phosphate esters, such as tricresyl phosphate (TCP), tributyl phosphate (TBP), triphenyl phosphate (TPP), etc., were initially used for aviation engines. Despite the health concerns that they pose [6,7], their use has expanded, and they are presently employed in a wide range of applications, including automotive engines [1].

Although environmental and health concerns place limitations on the amount of phosphorus in lubricants, the additives are expected to be utilized well into the future. Indeed, one common requirement for many systems is that newly developed lubricant additives must be compatible with 
existing phosphate esters additives, rather than outright replacing them. An appropriate strategy to limit their use levels while also retaining their function is to blend them with a synergistic and environmentally friendly additive. The overarching goal here is to add the synergistic additive without increasing the amount of phosphorus in the system until it is required to do so. This strategy would be completely practical to implement and applicable in various fields, such as aviation, automobiles, manufacturing, defense, etc., that implement a condition-based maintenance schedule [8].

Nanomaterials are emerging as environmentally friendly and effective additives for controlling friction and wear in various applications [9]. Several physical mechanisms such as change in friction mode (sliding to rolling), surface polishing/mending, reduction in cohesive forces via layered materials, etc., and chemical mechanisms, including material delivery/transfer for catalysis, tribofilm formation by tribochemical reactions, etc., have been identified as lubrication mechanisms for nanomaterials [10]. However, nanomaterials should also be able to withstand tough environments under EP conditions during boundary lubrication and the high operating temperatures of aircraft engines if they are to be successfully blended with currently used additives. This requires that nanomaterials have, among other properties, a high melting point, high hardness, and favorable chemical reactions to allow rigid bonding onto the surface, together with the anti-wear film produced by the phosphate ester [5].

Among the nanomaterials discovered and studied so far, nanodiamonds are the leading candidates from the point of view of hardness, high melting point, and the ability to withstand extreme environments. However, their impact on the performance of existing phosphate ester anti-wear additives is unknown. Several studies of nanodiamond additives have revealed their ability to improve tribological performance in various applications as well as various lubrication regimes including boundary lubrication [11-13]. However, their direct influence on the performance of existing additives has not been extensively studied to date. Since nanodiamonds have been reported to suppress reaction kinetics in certain systems by raising the activation energy for reactions [14], it is critically important to assess their direct effect on the reaction activities of existing anti-wear additives. Prior studies on various reaction mechanism paths through which phosphate esters form films on Fe surfaces have revealed that there is a requirement to overcome an activation energy to break either $\mathrm{P}-\mathrm{O}$ or $\mathrm{O}-\mathrm{C}$ bonds on the phosphate esters, depending on whether they are aliphatic or aromatic [15-17]. The activation energy can be attained with thermal energy, resulting in a thermal reaction film, or by other means, such as rubbing the contacts to form a tribofilm, etc. Among the various cases, the films formed are similar in chemistry, but the formation temperature varies significantly [18]. Nanodiamonds, as a proposed synergistic additive to phosphate esters, should clearly not have unfavorable effects on the reaction kinetics.

To explore this issue, we studied the effects of nanodiamonds on the formation of thermal reaction films on Fe and air baked Fe surfaces by TCP in a dibasic ester basestock. The TCP thermal reaction film formation temperatures in the presence and absence of nanodiamonds were recorded in-situ by monitoring of the reaction film formation on the surfaces using a quartz crystal microbalance (QCM) [19]. We recently reported the in-situ TCP thermal reaction film formation temperatures for Fe to be $210{ }^{\circ} \mathrm{C}$ [19]. Fe samples that were baked in air beforehand did not, however, react with TCP to form the thermal reaction film. It is therefore of interest to explore how the presence of nanodiamonds in the blend would influence whether or not thermal reaction films form on these substrates and whether the resulting reaction films, if present, are impacted by the nanodiamonds. The surface morphology, roughness, and thickness of the thermal reaction films that did form were studied with an atomic force microscope (AFM), and their chemical compositions were studied with Electron Dispersive X-ray Spectroscopy (EDS). The TCP-derived films were regarded as anti-wear films, rather than materials having the property of decreasing the coefficient of friction. Since the QCMs are fragile, the thermal reaction films formed on the QCM substrates could not withstand the rigorous tribo-testing procedure that is regularly performed for such anti-wear films. We, therefore, as a simple gauge, employed a basic friction measurement approach to provide information on the coefficient of sliding friction at the dry solid-solid contact between the thermal reaction films and 
the stainless-steel balls. The contact angles between the thermal reaction films and the base oil were measured to examine any changes in the solid-liquid wetting property of the surfaces after the TCP uptake. Change in contact angles are associated with changes in the physical and chemical properties of the surfaces after formation of the thermal reaction films. In particular, it is important to identify changes in the slip condition at the QCM's solid-liquid interface [20].

\section{Materials and Methods}

Diisodecyl Adipate (Sigma-Aldrich Co. LLC, St. Louis, MO, USA) was used as the base oil. The TCP (Sigma-Aldrich Co. LLC) was of a technical grade and was a mixture of isomers. One-inch diameter, $5 \mathrm{MHz}$, AT-cut QCMs with liquid-facing surface electrodes comprised of Fe (INFICON Inc., East Syracuse, NY, USA, P/N 149252-1) were employed for QCM measurements. These Fe-coated QCMs consisted of $800 \mathrm{~nm}$ Fe films deposited onto $60 \mathrm{~nm} \mathrm{Cr}$ adhesion layers deposited onto the QCM surfaces. The air baked Fe samples were obtained by heating the Fe-coated QCMs in air. Nanodiamonds with $30 \mathrm{~nm}$ diameters dispersed in oil at a concentration of $1 \mathrm{wt} \%$ were obtained from Adamas Nanotechnologies. The nanodiamond dispersion was diluted to the desired concentration in the base oil and then used for further measurements.

The QCM measurement techniques employed here have been previously described in great detail $[19,21]$. The QCM crystals were housed in a custom made holder and then immersed in oil containing either TCP or a TCP-nanodiamond blend. The holder was then mounted within a temperature-controlled chamber and heated from room temperature to $\sim 240{ }^{\circ} \mathrm{C}$ over $2 \mathrm{~h}$. Data were collected using a QCM100 (Stanford Research Systems, Sunnyvale, CA, USA) system, comprised of an electronic oscillator and a controller [22]. The frequency and conductance of the crystal were measured by a frequency counter (HP 53181A Frequency Counter, Palo Alto, CA, USA) and a multimeter (TEKTRONIX, INC., Beaverton, OR, USA, Keithley 2000 Series), respectively. The frequency shifts of the QCM provided information on film uptake, liquid viscosity, and film stress. Conductance measurements provided information on dissipative losses associated with the drag forces of the surrounding liquid and/or losses within the reaction film itself. A second multimeter measured the resistance of an RTD attached to the holder. A LabVIEW program (National Instrument, Austin, TX, USA, LabVIEW 7.1) recorded the frequency and conductance of the QCM and resistance of the RTD at $5 \mathrm{~s}$ intervals. The conductance, initially measured in volts, was converted to the motional resistance of the QCM using the relationship, $R=10^{(4-V / 5)}-75$ [22], where $V$ is the conductance in volts and $R$ is the motional resistance of QCM in Ohms. The RTD resistance was converted to temperature by employing the Callendar-Van Dusen equation [23].

To correct the data for the temperature effects, the crystal was first heated in air to obtain the temperature dependence of the frequency and the motional resistance response. The crystal was then held at $225^{\circ} \mathrm{C}$ for $2 \mathrm{~h}$, allowing the iron to oxidize, and is referred to as the "baked Fe" sample herein. Both the "baked Fe" samples and fresh (unheated) samples of Fe were employed for the TCP thermal reaction film monitoring studies. Since bare metal surfaces always have a few layers of chemisorbed oxide, a native oxide was presumed to be present on the unheated Fe samples. Thermal reaction film formation temperatures were recorded in-situ by monitoring the reaction film formation on the Fe and "baked Fe" by immersing the QCM samples in the basestock with 5\% TCP in the absence or presence of $0.1 \mathrm{wt} \%$ nanodiamonds and then heating them from room temperature to $\sim 240{ }^{\circ} \mathrm{C}$. QCM responses were then corrected for temperature effects to isolate the effects of oil and thermal reaction films (if formed).

After the QCM measurements, the crystals were cleaned with iso-propanol to remove excess oil from the surface. The surface morphology, roughness, and thickness of the thermal reaction films were then studied with an AFM (MFP 3D, Asylum Research, an Oxford Instruments Company, Santa Barbara, CA, USA). Silicon tips were used to image the surfaces in tapping mode. Images with $512 \times 512$ pixels were recorded with a speed of 1 line per second. The scan sizes ranged from 2 to $6 \mu \mathrm{m}$. Their roughness 
was characterized by the root mean square (rms) roughness value, $\sigma=\left(\frac{\sum\left\{h\left(x_{i}, y_{i}\right)-\bar{h}\right\}^{2}}{n-1}\right)^{\frac{1}{2}}$, and also, the average roughness value, $R_{a}=\left(\frac{\sum\left|h\left(x_{i}, y_{i}\right)-\bar{h}\right|}{n}\right)$, which provide a measure of the magnitude of variation between the surface height profiles. Surface roughness was also characterized by a third parameter, the self-affine fractal roughness exponent $\mathrm{H}$, which reflects the nature of the texture of surface irregularities on the surfaces. For a self-affine surface, $\mathrm{H}$ is related to $\sigma$ and scan size $(L)$ as $\sigma \propto L^{H}$. It can be obtained from the slope of a linear fit of $\log (\sigma)$ vs $\log (L)$ plot for the scan sizes below the saturation length [24]. The fractal dimension, $D=3-\mathrm{H}$, for the QCM surface electrodes before and after the TCP uptake were calculated after estimating the values of $\mathrm{H}$ for the respective surfaces. A surface with $D=2$ is a perfect 2D surface, while a $D$ value higher than 2 indicates surfaces with more texture that deviate from a $2 \mathrm{D}$ plane. The thicknesses of the thermal reaction films were measured by calculating the differences in AFM height profiles measured across the film-surface boundary after etching the thermal reaction film with $0.3 \mathrm{M}$ oxalic acid (Sigma-Aldrich Co. LLC.). The chemical compositions of the thermal reaction films were studied with EDS (JSM-6010LA Analytical Scanning Electron Microscope, JEOL USA Inc., Peabody, MA, USA). The measurements were performed with a $20 \mathrm{kV}$ acceleration voltage, a $10 \mathrm{~mm}$ working distance, and with $100 \times$ magnification. The contact angles of the base oil on the QCM electrode surfaces before and after the TCP uptake were measured using the drop shape analysis technique [25].

As mentioned earlier, the thermal reaction films formed on the QCM substrates could not be employed for a rigorous tribo-testing procedure because of the fragility of the quartz substrates. Therefore, the coefficients of sliding friction at the dry solid-solid contact between the thermal reaction film and 304 stainless-steel ball bearings were measured by a conventional Pasco laboratory instrument (Pasco Scientific, Roseville, CA, USA). Seven stainless-steels balls of 5/32' diameter were glued onto a weight (in hexagonal close packed configuration). The weight was then placed on the surface electrode of a QCM glued on a platform, as shown in the Figure 1. The weight was then pulled by another weight with a lightweight cord tied to it while measuring the force continuously until a constant lowest possible relative speed was maintained between the QCM surface and the sliding weight. The coefficient of sliding friction was then furnished from the applied force and the normal force.

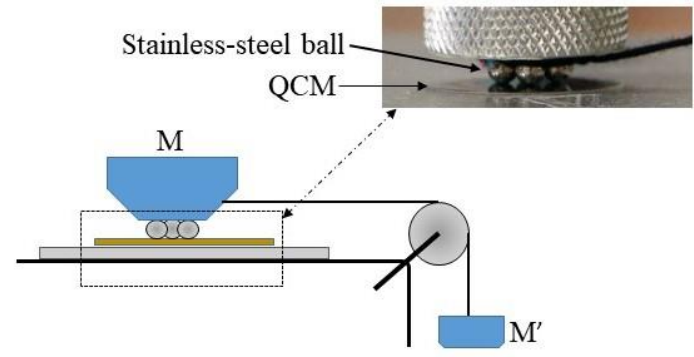

Figure 1. Experimental setup for the measurement of coefficient of friction (see text for detail).

\section{Results}

The results are presented in five sub-sections. In the first section, the in-situ QCM measurements on Fe and baked Fe electrodes immersed in 5\% TCP in basestock in the absence and presence of 0.1 wt \% nanodiamonds are presented, showing the associated features that are indicative of the formation of thermal reaction films. All of the QCM measurements depicted represent the average response from 2-4 measurements, and very little difference was observed from one run to another. The changes in QCM frequency $(\delta f)$ and motional resistance $(\delta R)$ reported were measured with respect to their corresponding initial values in air at room temperature. The AFM measurements of film topology and contact angle measurements are presented in Section 3.2, while the film thickness measurements are 
reported in Section 3.3. EDS measurements of chemical composition of the thermal reaction films are presented in Section 3.4, followed by a report of the friction measurements in Section 3.5.

\subsection{QCM Measurements}

The $f$ and $R$ responses of QCMs with Fe surfaces for room temperature to $240{ }^{\circ} \mathrm{C}$ in basestock with $5 \%$ TCP and with and without $0.1 \%$ nanodiamond are shown in Figure $2 a, b$, respectively. The QCM responses for both conditions (below the reaction film formation temperatures) followed the trend for the liquid loading with variations in $f$ and $R$ with temperature being primarily due to the temperature dependent viscosity and density of the oil. As reported earlier [19], the sharp increases in $\delta f$ and $\delta R$ observed at $210{ }^{\circ} \mathrm{C}$ for the $5 \%$ TCP on Fe are attributable to the formation of a TCP thermal reaction film on the Fe surface electrode on the QCM. This feature was, however, shifted to a higher temperature of $228^{\circ}$ for the TCP + nanodiamond blend. Optical images of the QCMs revealed that the thermal reaction film is distributed homogeneously over the entire surface in the absence of nanodiamonds, while in the presence of nanodiamonds, it exhibits inhomogeneity which is attributable to an inhomogeneous distribution of nanodiamonds imbedded within the film.

The $f$ and $R$ responses of QCMs with baked Fe surfaces are shown in Figure 3a,b, respectively. The QCM responses for baked Fe in the absence of nanodiamonds exhibited a similar trend for the liquid loading with variations in $f$ and $R$ with temperature associated with the temperature dependent viscosity and density of the oil. No characteristic feature was observed in the absence of nanodiamonds: As we have previously reported, thermal reaction films do not readily form on the thick oxide layer formed by baking the Fe sample in air. However, in the presence of nanodiamonds, the feature associated with the formation of a TCP thermal reaction film was observed at $228{ }^{\circ} \mathrm{C}$. Changes in frequency and motional resistance were also observed in the presence of nanodiamonds, starting at around $190{ }^{\circ} \mathrm{C}$. The optical images of the QCM for the absence of nanodiamonds showed some discoloration but no film on the surface, while for the presence of nanodiamonds, the film appeared visually similar to that on the Fe surface, as shown in Figure 2.
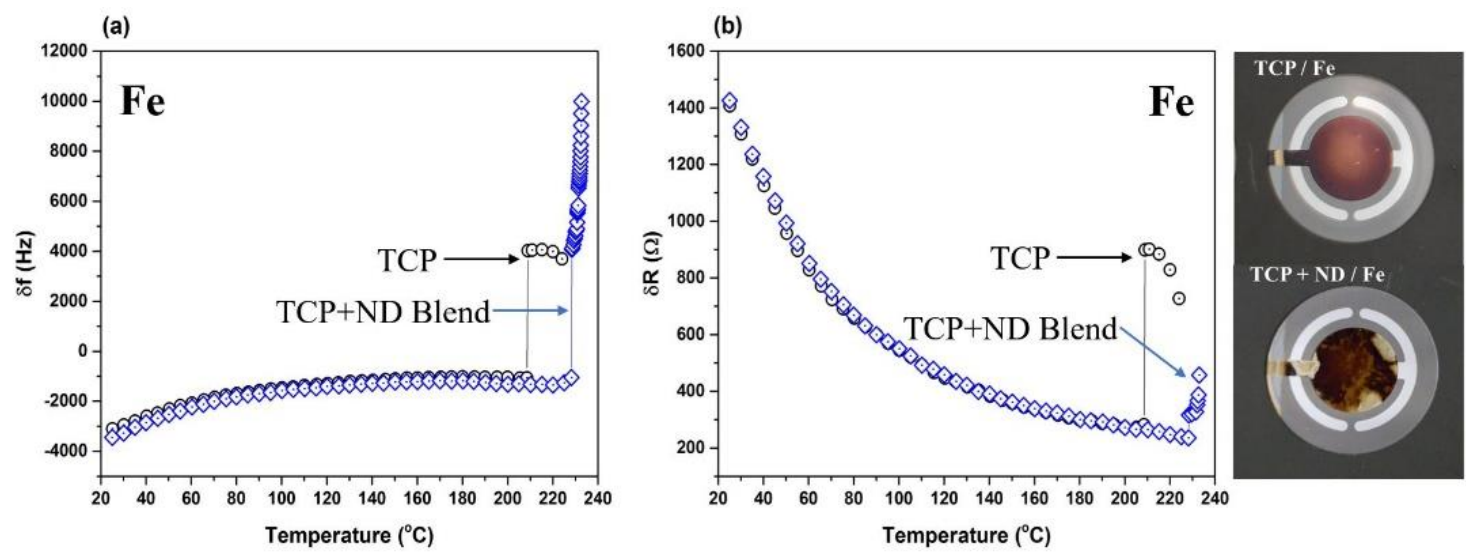

Figure 2. Shifts in (a) frequency and (b) motional resistance of quartz crystal microbalances (QCMs) with Fe surface electrodes in basestock with 5\% tricresyl phosphate (TCP) and with (diamonds) and without (circles) $0.1 \%$ nanodiamond. The optical images of these QCMs are shown on the right-hand-side. 

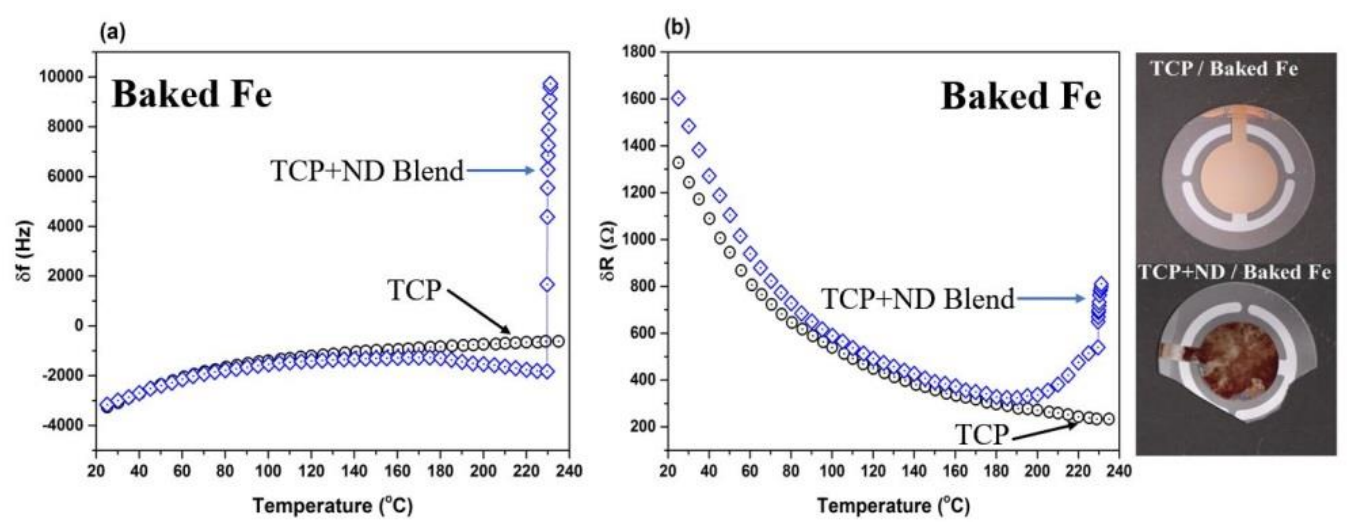

Figure 3. Shifts in (a) frequency and (b) motional resistance of QCMs with Fe surface electrodes baked in air, in basestock with 5\% TCP and with (diamonds) and without (circles) $0.1 \%$ nanodiamonds. The optical images of these QCMs are shown on the right-hand-side.

\subsection{AFM Surface Morphology, Roughness, and Contact Angle Measurements}

Figure 4 shows the Fe and baked Fe surfaces after the TCP uptake in the absence and presence of nanodiamonds. The baked Fe surface without the nanodiamonds showed no film on it, and the surface appeared similar to that before the TCP uptake (see reference [19]). The other three film forming surfaces showed similar morphologies. The films formed in the presence of nanodiamonds were expected to show the presence of nanodiamonds on their surfaces; however, we could not confirm this from the AFM measurements on these films. It is plausible that the nanodiamonds were not present on the top of the film to interact with the AFM probe. The values of $\sigma, R_{a}, D$, and the contact angles between the base oil and the various surfaces are shown in Figure 5. The thermal reaction films for the three film forming systems were observed to have similar roughnesses $\left(\sigma\right.$ or $\left.R_{a}\right)$ with values smaller than those of the corresponding surfaces before the film formation. The values of $D$ for the thermal reaction films formed in the presence of nanodiamonds were significantly higher, implying a slightly more jagged surface texure for these films relative to the others. Despite a significant change in texture, the presence of nanodiamonds in the oil did not increase the magnitude of either $\sigma$ or $R_{a}$ for the films, and, as will be described in a later section, the nanodiamonds were not observed to be protruding above the top of the films. The contact angle for the thermal film formed on the Fe surface was slightly lower when nanodiamonds were present which is potentially beneficial. No such comparison could be made for the thermal films formed on the baked Fe substrates, since the films did not form on them in the absence of ND. 

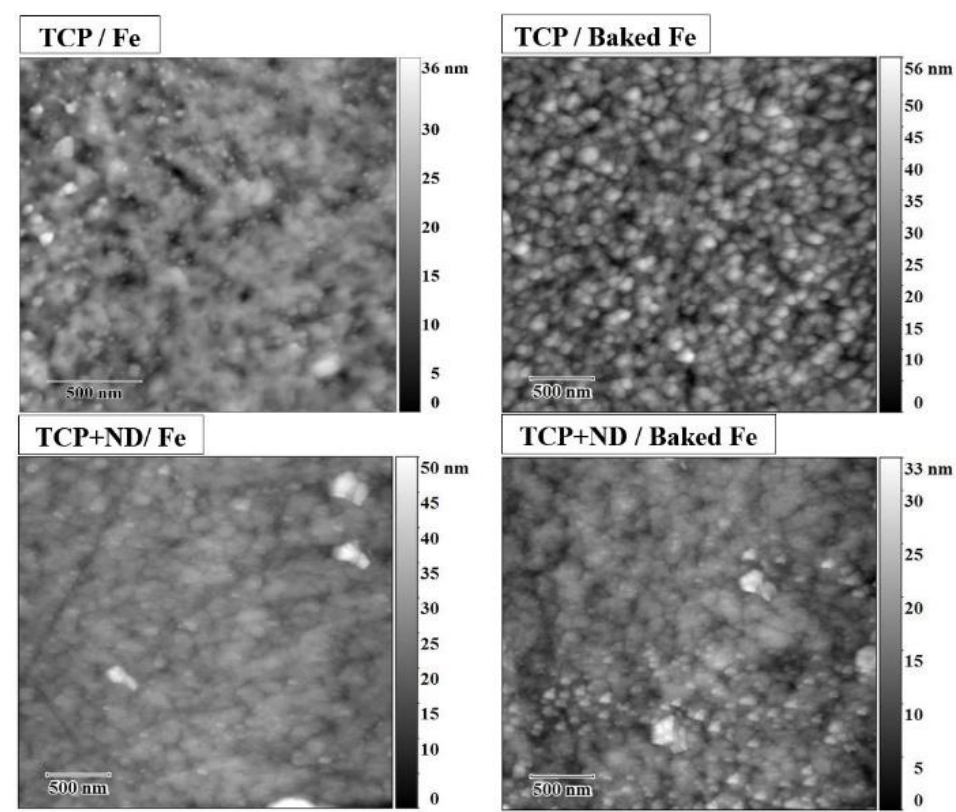

Figure 4. AFM surface morphologies of the surface or reaction films on the QCM surface electrodes after various experimental conditions.
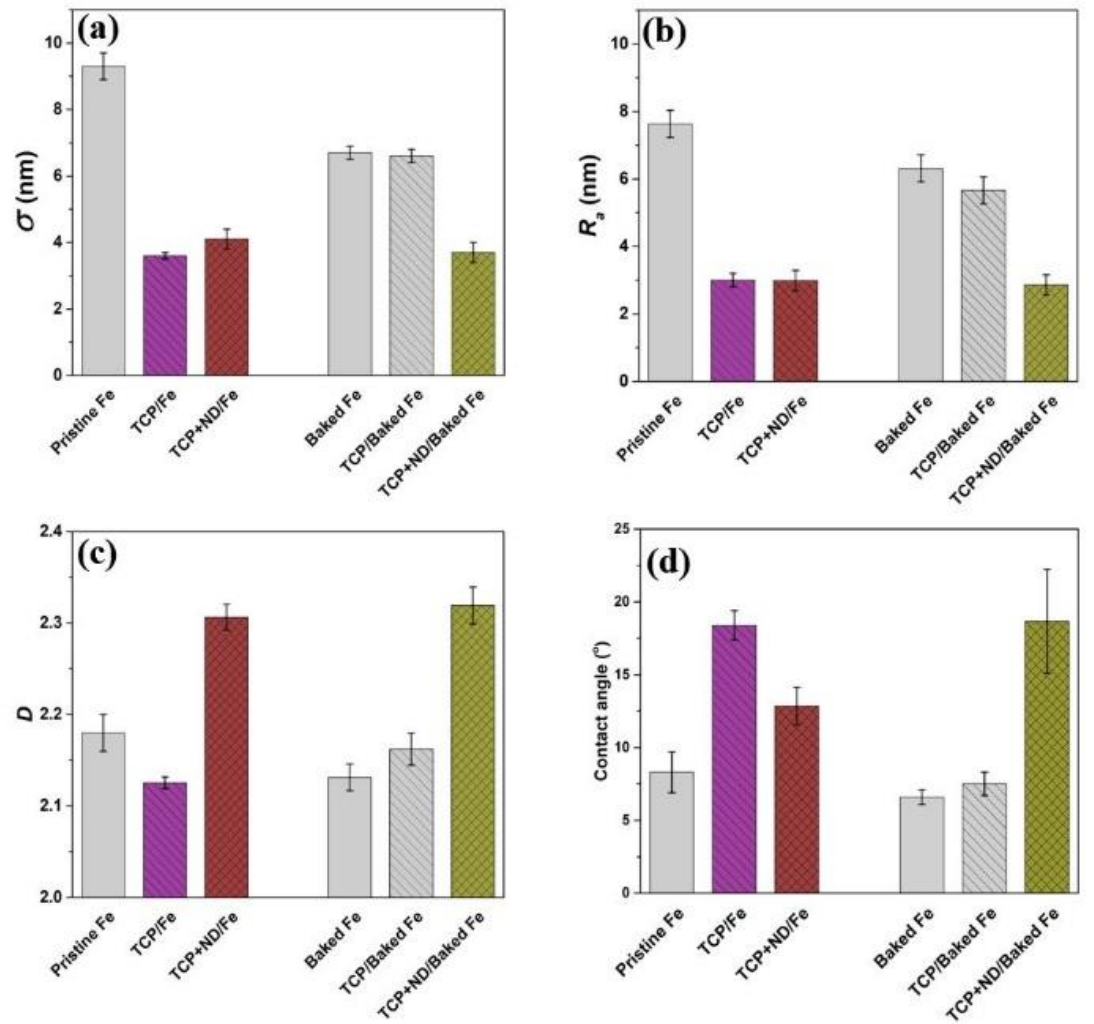

Figure 5. The roughness parameters $(\mathbf{a}) \sigma,(\mathbf{b}) R_{a}$ and (c) $D$ of the various samples, and (d) the contact angles relative to the base oil. The baseline for $D$ is 2 , which is its lowest value that is associated with a perfect 2D plane. The reported values of $\sigma$ and $R_{a}$ were measured over a surface area larger than the saturation length for the respective surfaces. 


\subsection{Measurement of Film Thickness}

We measured the film thickness ex-situ by etching through the thermal reaction films with a pin dipped in oxalic acid and then measuring their thicknesses at the film-surface boundaries with AFM. Oxalic acid is particularly effective for such measurements as it has minimal impact on the Fe substrate underneath the film. We have previously reported that the thickness of the thermal reaction film on Fe surface in the absence of nanodiamonds can be observed in the range of 60-80 nm, and during the measurement, we noted that the thermal reaction films on Fe surfaces were readily removed from the surfaces with oxalic acid [19]. However, a similar protocol was not applicable for the thermal reaction films formed on Fe surfaces in the presence of nanodiamonds, because these films were relatively stronger. The acid etching could only remove the top layer from this relatively stiffer film, so an additional mechanical force from the pin was required to remove the film completely. Figure 6 shows the groove formed on the thermal reaction film by the pin. On either side of the groove, the top layer from the thermal reaction film was etched away which fortuitously uncovered the nanodiamonds embedded into the film. The expanded view of the selected section of thermal reaction film revealed many small, circular protrusions with diameters in the range of 20-50 nm and heights of up to $20 \mathrm{~nm}$ (ibw format file of the AFM image is available in the Supplementary Material). These were the nanodiamonds that were embedded into the thermal reaction film formed in the presence of nanodiamonds. Since these nanodiamonds partially protruded out of the etched film, it was estimated that, at minimum, a $20 \mathrm{~nm}$ thick top layer film must have been etched away. The measured height profiles across the groove showed that the depth of the groove was in the range of $45-60 \mathrm{~nm}$. Therefore, the total thickness of the thermal reaction film ranged from 65 to $80 \mathrm{~nm}$. This film thickness for the thermal reaction film formed on Fe surfaces in the presence of nanodiamonds was same as the thickness of the film formed in the absence of nanodiamonds. This revealed that the thickness and the average rate of formation of the thermal reaction film (estimated to be about $2.3 \mathrm{~nm} / \mathrm{min}$ [19]) was unaffected by the presence of nanodiamonds, though the nanodiamonds were found to be embedded into the film, making the film stiffer, as gauged by the QCM response data. The estimated quantity of nanodiamonds in the film, based on Figure $6 \mathrm{c}$, was $9.7 \times 10^{19}$ particles $/ \mathrm{m}^{3}$, which is about five times higher than the concentration of $1.8 \times 10^{19}$ particles $/ \mathrm{m}^{3}$ in the original solution. This is not unexpected given that the total volume of the TCP + ND blend used in the experiments was about $5 \mathrm{~mL}$, and the distribution of nanodiamonds in the film was inhomogeneous which may have resulted in the five-fold increase in concentration of some sections within the film.
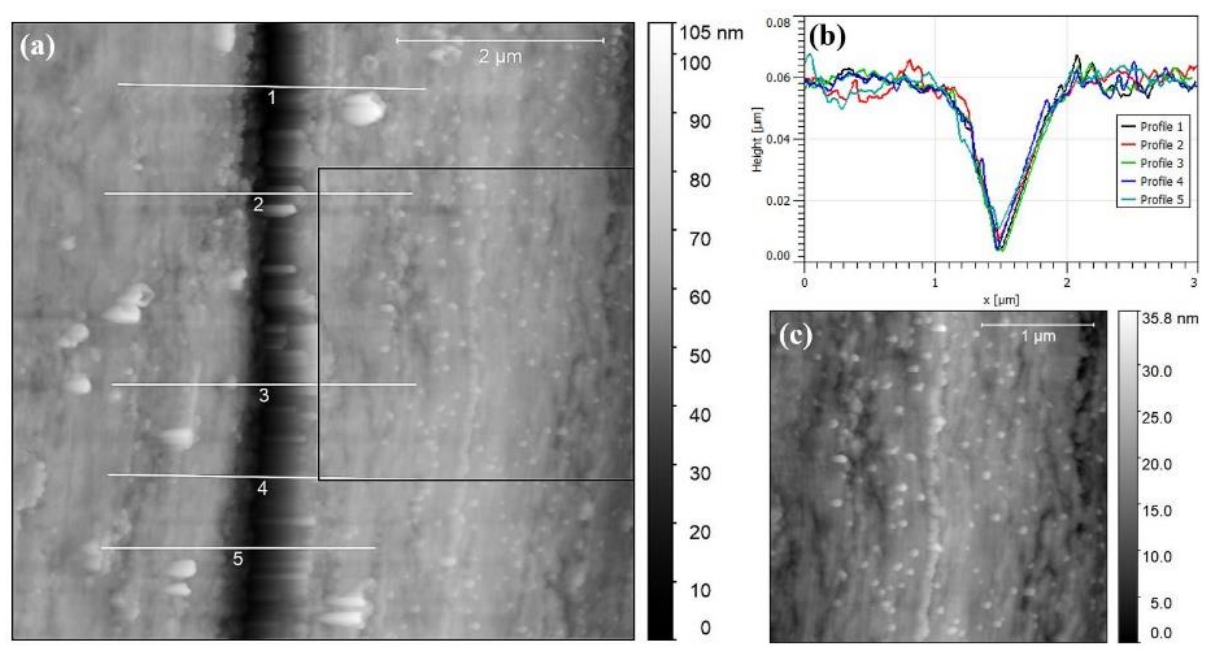

Figure 6. (a) AFM image at the film-surface boundary after the thermal reaction film on Fe surfaces in the presence of nanodiamonds had been etched with a pin dipped in $0.3 \mathrm{M}$ oxalic acid. (b) The height profiles (1-5) along the lines drawn across the boundaries (in (a)). (c) The expanded view of the selected section of thermal reaction film (in (a)). 


\subsection{EDS Measurements of Film Chemical Compositions}

The chemical compositions of the Fe and baked Fe surfaces heated in base oil or in 5\% TCP in base oil in the presence and absence of nanodiamonds obtained by the EDS are shown in Table 1. We note that the Cr metal used as an adhesive layer between the quartz disc and the Fe sensing electrodes was also present in the EDS measurements as well as silicon attributable to the quartz substrates. This is to be expected, given the penetration depth of the $20 \mathrm{kV}$ accelerating voltage employed for the measurements. Figure 7 is a graphical representation of the selective elements $(\mathrm{P}, \mathrm{O}$ and $\mathrm{C})$ from Table 1 . The thermal reaction films formed on the $\mathrm{Fe}$ (with and without nanodiamonds) and baked Fe (with nanodiamonds) revealed the presence of phosphorus, whereas the surfaces with no reaction films had no phosphorus content. The TCP thermal reaction films have been reported in prior literature $[3,19,26]$ to comprise inorganic phosphates, consistent with the increase in $\mathrm{P}$ and $\mathrm{O}$ content but not the significant increase in $\mathrm{C}$ content. With the presence of nanodiamonds, the thermal reaction films on both the Fe and baked Fe surfaces had significantly higher levels of $C$ compared to films without nanodiamonds. This increase in $C$ is attributed to the nanodiamonds being embedded into the thermal reaction films. The $\mathrm{O}$ content of the film formed on the baked Fe surfaces weres much higher than the films formed on the Fe surfaces. This is consistent with the fact that the formation of the thermal reaction film on the baked Fe surfaces in the presence of nanodiamonds followed a different mechanism path, as discussed later in the discussion section.

Table 1. Chemical compositions of the various samples, obtained by EDS. The values shown represent the average of the measurements at multiple locations on the samples.

\begin{tabular}{ccccccc}
\hline \multirow{2}{*}{ Samples } & \multicolumn{6}{c}{ Chemical Composition (mol \%) } \\
\cline { 2 - 7 } & $\mathbf{C}$ & $\mathbf{O}$ & $\mathbf{S i}$ & $\mathbf{P}$ & $\mathbf{C r}$ & $\mathbf{F e}$ \\
\hline Base Oil/Fe & 11.2 & 7.8 & 1.3 & 0.0 & 1.5 & 78.2 \\
TCP/Fe & 13.0 & 12.6 & 1.0 & 0.4 & 1.2 & 71.8 \\
ND + TCP/Fe & 18.0 & 12.8 & 1.1 & 0.3 & 1.1 & 66.7 \\
TCP/Baked Fe & 9.8 & 7.6 & 2.2 & 0.0 & 1.9 & 78.5 \\
ND + TCP/Baked Fe & 20.0 & 18.0 & 1.2 & 0.3 & 1.0 & 59.5 \\
\hline
\end{tabular}

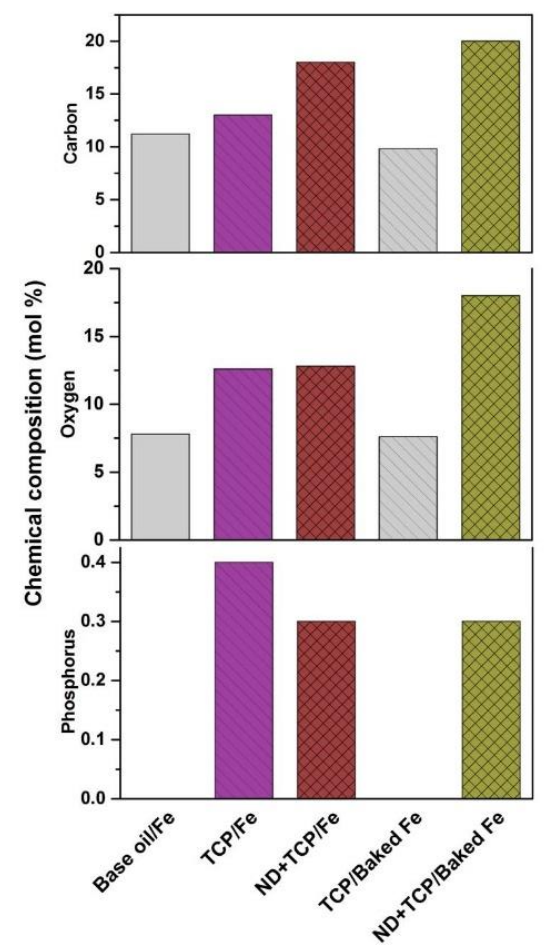

Figure 7. The chemical compositions ( $\mathrm{mol} \%)$ for the selective elements ( $\mathrm{P}, \mathrm{O}$ and $\mathrm{C})$ shown in Table 1. 


\subsection{Measurement of Friction Coefficient}

The coefficients of friction during dry solid-solid contact between various substrates or thermal reaction films and stainless-steel balls are shown in Figure 8. The coefficient of friction for the thermal reaction film on Fe without nanodiamonds had the lowest value; however, the presence of nanodiamonds increased it to a slightly higher value. This result is consistent with the observed contact angles for these thermal reaction films. On the other hand, the coefficient of friction measured for the thermal reaction film on baked Fe in the presence of nanodiamonds was not observed to have similar values to the films formed on Fe surfaces. This is notable because all of the thermal reaction films were expected to have a similar coefficient of friction on the basis of the roughness and contact angle measurements. However, during the friction measurements, we observed that the thermal reaction film formed on baked Fe surfaces in the presence of nanodiamonds was not very durable. The film was scratched off rather easily, exposing the substrate underneath it, and the surface was seen to have wear marks, even after a couple of measurement trials. For this reason, the coefficient of friction for this surface may have remained higher compared to the those for the baked Fe surfaces without the film. For comparison, the optical images of the thermal reaction films formed on the Fe and baked Fe surfaces in the presence of nanodiamonds after the friction measurements are shown in Figure 8. The thermal reaction film on the Fe surface was observed to have excellent durability with no visible marks, while the thermal reaction film on the baked Fe surface seemed to wear off and have scar marks over its entire surface.

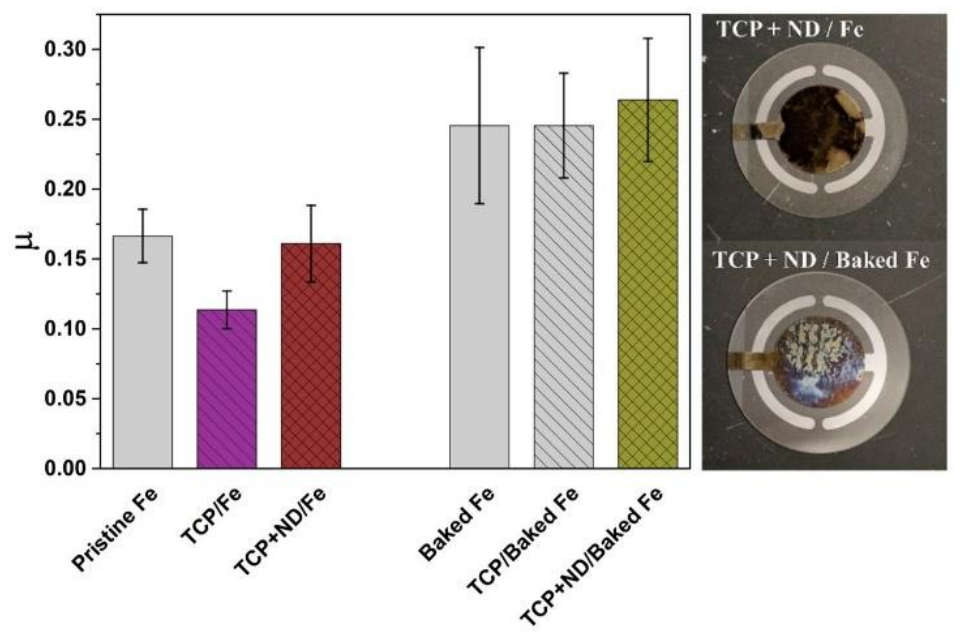

Figure 8. The coefficients of sliding friction measured during dry solid-solid contact between surfaces of various QCM samples and stainless-steel balls. The QCM images (on the right-hand-side) after the friction measurements showing the conditions of the thermal reaction films on the Fe and baked Fe surfaces formed in the presence of nanodiamonds are shown.

\section{Discussion}

The nanodiamonds were observed to influence the formation of TCP thermal reaction films on both Fe and baked Fe surfaces. It is important to identify whether or not these effects are favorable. Figure 9 shows the QCM responses of all film-forming systems presented in Figures 2 and 3. TCP was observed to form a thermal reaction film on baked Fe surfaces in the presence of nanodiamonds which otherwise did not form the film. This is a notable characteristic of nanodiamonds. The changes in $f$ and $R$ in the presence of nanodiamonds observed on baked Fe starting at $190{ }^{\circ} \mathrm{C}$ suggested the adsorption of mass onto the surface. This is likely to be due to the nanodiamonds either making their way to the inner layers of the oxide film or causing wear to the oxide layers, thus allowing the TCP molecules to absorb into the inner layers of the oxide surface. This mechanism is conceivable since the oscillation of QCM at a frequency of $5 \mathrm{MHz}$ would produce a maximum oscillating surface speed 
in the order of $10 \mathrm{~cm} / \mathrm{s}$, and nanodiamonds have been reported to have these characteristics under such conditions [27]. The TCP molecules making their way to the Fe through the oxide layers then formed a thermal reaction film on the surface when the activation energy reached a temperature of $228^{\circ} \mathrm{C}$. This thermal reaction film was expected to have a higher oxygen content which was, indeed, observed during the EDS measurements. In the presence of nanodiamonds, the formation of the thermal reaction films was observed on both Fe and baked Fe surfaces at the same temperature of $228{ }^{\circ} \mathrm{C}$ (within the experimental error) which is higher by $18{ }^{\circ} \mathrm{C}$ than that observed on Fe surfaces in the absence of nanodiamonds. This result is not unexpected, because it was anticipated that the nanodiamonds would raise the activation energy in such reactions. The increase in activation energy is not, however, highly disadvantageous, as the observed increase in reaction temperature was modest and readily achievable in actual applications.

(a)

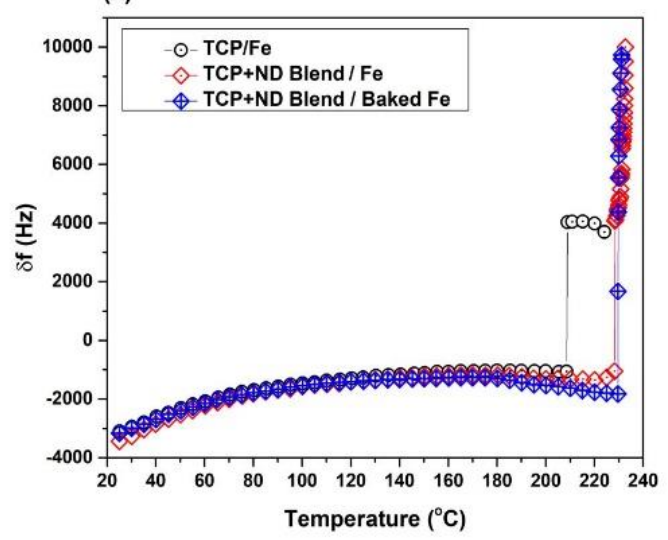

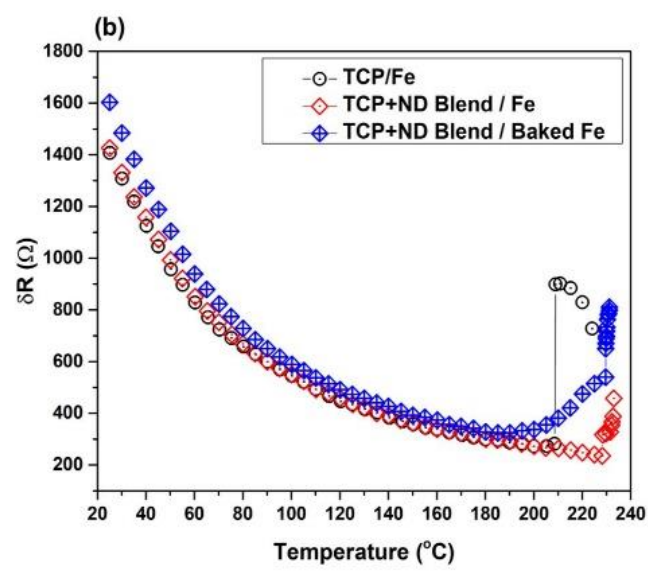

Figure 9. Shifts in (a) frequency and (b) motional resistance for the film-forming systems shown in Figures 2 and 3.

The change in motional resistance observed for formation of the thermal reaction film is associated with changes in the QCM's dissipative properties, such as a change in the slip condition at the solid-liquid boundary, an increase in energy dissipation within the viscoelastic film on the QCM's surface, a change in surface roughness, etc. The observed shift in $R$ was not in the direction expected from the smoother films and/or surfaces with higher contact angle values (c.a. Figure 5) [20,28]. Therefore, the higher increase in $R$ during the film formation on Fe surfaces in the absence of nanodiamonds suggested that this film is relatively softer or more viscous compared to the films formed in the presence of nanodiamonds [29]. This implies that the films formed in the presence of nanodiamonds are more rigid which is to be expected if the nanodiamonds are embedded into the film, as they are potentially capable of exhibiting more outward stress.

The increase in frequency observed during the formation of thermal reaction films is in fact consistent with stress developing within the film. The rigidly-bonded thermal reaction film on the surface acts to stretch the surface and causes stress on the crystal. The positive $f$ shift for the AT-cut quartz indicates that the tensile stress was isotropic and outward. The average stresses on the crystal and the thermal reaction film on the Fe surface in the absence of nanodiamonds (corresponding to the associated $\delta f \approx 5 \mathrm{kHz}$ ) have been reported to be $T_{Q} \approx 40 \mathrm{MPa}$ and $T_{f} \approx 200 \mathrm{GPa}$, respectively [19]. The value of this internal stress for the thermal reaction film is in the same order of the effective modulus as the TCP tribofilms, and the stress on the crystal is in the same order as the shear modulus of quartz [3]. However, the frequency shifts associated with the film formation in the presence of nanodiamonds were significantly higher compared to the film formed in the absence of nanodiamonds (c.a. Figure 9). These higher frequency shifts are consistent with the idea that increased stress is present when nanodiamonds are present in the film. The average stresses on the crystals and the thermal reaction films formed on the Fe and baked Fe surfaces in the presence of nanodiamonds (corresponding 
to the associated $\delta f>10 \mathrm{kHz}$ ) would be higher than their corresponding elastic moduli. These stresses greatly inhibit the ability of the crystal to oscillate. During the measurements, the crystals indeed stopped oscillating about 2-3 min after the film formation in the presence of nanodiamonds. The $\delta f$ and $\delta R$ data shown in Figure 3, Figure 4, and Figure 9 for both surfaces in the presence of nanodiamonds were recorded until the crystals oscillated after the film was formed. It is expected that the $\delta f$ would have increased further until it plateaued once the reaction was nearly exhausted, as seen in the case of the Fe surfaces without nanodiamonds. These steeply increasing values of $\delta f$ in the presence of nanodiamonds meanwhile confirmed that although these nanodiamonds increase the thermal reaction film formation temperature by raising the activation energy, the reaction rate may not necessarily be slower, as the reaction for the phosphate esters, being exothermic, readily provides the required activation energy to continue the reaction once it is initiated $[15,16]$.

The films formed in the presence of nanodiamonds were observed to be stiffer and exhibited higher levels of internal stress. The internal stress of the reaction films is an integral part of their performance, since it counteracts external load forces, thus enhancing the load-bearing capacity of the substrate [30]. The nanodiamonds also changed the texture of the thermal reaction films (c.a. Figure $5 \mathrm{c}$ ) while increasing their wettability with the lubricant (c.a. Figure 5d). These effects are beneficial, especially in extreme pressure conditions, whence the lubricants can easily be squeezed out from the contact at the surfaces with lower wettability, lowering the resistance to scuffing [31,32].

The thermal reaction film on the baked Fe surfaces in dry conditions in an ambient environment was observed not to have the signature characteristics of a typical anti-wear film. One plausible reason for this could be that the film formed on baked Fe surfaces with a different mechanism and has a higher oxygen content, as observed in the EDS measurements. The higher amount of oxygen probably has an effect on the strength of the film during dry contact, because the oxygen can absorb the moisture/humidity from the ambient air rather easily and deteriorate the film strength [33]. A thorough investigation is necessary to confirm this suggestion and also, to find other probable causes. While the nanodiamonds did not favor the dry contact on the baked Fe surfaces, it might be beneficial in other situations, especially under mixed lubrication on surfaces with higher oxide contents. Having the film is always more beneficial than having a bare surface. In real applications, in oil environments, the nanodiamonds would be able to perform the special role of enabling film formation and replenishing it continuously from the fresh supply of oil while the tribo-pairs are in action. The rate of film formation, however, must not be exceeded by the wear rate [34].

\section{Conclusions}

The impacts of nanodiamonds on the reaction activity and performance of a phosphate ester additive, tricresyl phosphate, were studied. In summary, the effects of nanodiamonds were observed to be synergistic with the TCP. The nanodiamonds enabled the formation of a reaction film on baked Fe which otherwise did not form the reaction film. They were observed to be embedded into the film and made the film stiffer, and, as indicated by the QCM data, the films with nanodiamonds were observed to have higher levels of internal stress. The internal stress on the reaction films themselves counteracts the forces of external loads, thereby enhancing the load-bearing capacity of the substrate [30]. For the Fe surfaces, reaction films with nanodiamonds were observed to be more durable. These observations reveal that the nanodiamonds constructively blend with TCP, resulting in more robust thermal reaction films. While the nanodiamonds delayed the formation of thermal reaction film by a modest amount, the rate of formation and the thickness of the thermal reaction film exhibited few effects. The tribo-films at the rubbing contacts were formed at lower temperatures than the temperature of the formation of the thermal reaction. Under such conditions, the activation energy is attained by several mechanisms, including flash point temperatures at contacts, tribo-emission processes and stress-promoted thermal activation, etc. [35] The effects of nanodiamonds on tribo-film formation by phosphate ester additives could be readily studied, and a rigorous tribo-testing procedure under EP conditions could be performed. The observations of the present study provide evidence 
that the nanodiamonds are likely to have similar synergistic effects on these mechanisms during the formation of the tribo-films and their tribological properties, and it also provides valuable information for numerical studies.

Supplementary Materials: Supplementary Materials are available online at http:/ /www.mdpi.com/2075-4442/ 6/2/56/s1.

Author Contributions: Conceptualization, B.A. and J.K.; methodology, B.A. and K.S.A.; validation, J.K.; formal analysis, B.A. and J.K.; investigation, B.A., K.S.A., B.T., and T.N.P.; data curation, B.A., K.S.A., B.T. and T.N.P.; writing—original draft preparation, B.A.; writing—review and editing, J.K.; visualization, B.A. and J.K.; supervision, J.K.; project administration, J.K.; funding acquisition, J.K.

Funding: This research was funded by the US National Science Foundation grant number DMR1535082.

Acknowledgments: We acknowledge Donald W. Brenner for project direction under the funding support DMR1535082. We are thankful to Adamas Nanotechnologies for providing the nanodiamond sample. This work was performed, in part, at the Analytical Instrumentation Facility (AIF) at North Carolina State University, which is supported by the State of North Carolina and the National Science Foundation (award number ECCS-1542015). The AIF is a member of the North Carolina Research Triangle Nanotechnology Network (RTNN), a site in the National Nanotechnology Coordinated Infrastructure (NNCI).

Conflicts of Interest: The authors declare no conflict of interest.

\section{References}

1. Johnson, D.; Hils, J. Phosphate Esters, Thiophosphate Esters and Metal Thiophosphates as Lubricant Additives. Lubricants 2013, 1, 132-148. [CrossRef]

2. Fujita, H.; Spikes, H.A. The formation of zinc dithiophosphate antiwear films. Proc. Inst. Mech. Eng. Part J J. Eng. Tribol. 2004, 218, 265-278. [CrossRef]

3. Hashimoto, C.; Okubo, H.; Tadokoro, C.; Sasaki, S. Correlation between Nano-Mechanical and Macro-Tribological Properties of Tribofilms Derived from Organic Phosphoric Additives. Tribol. Online 2016, 11, 632-638. [CrossRef]

4. Johnson, D.; Bachus, M.; Hils, J. Interaction between Lubricants Containing Phosphate Ester Additives and Stainless Steels. Lubricants 2013, 1, 48-60. [CrossRef]

5. Johnson, D.W. Lubricants for Turbine Engines. In Recent Progress in Some Aircraft Technologies; InTech: Vienna, Austria, 2016.

6. Michaelis, S. Contaminated aircraft cabin air. J. Biol. Phys. Chem. 2011, 11, 132-145. [CrossRef]

7. Ramsden, J.J. On the proportion of ortho isomers in the tricresyl phosphates contained in jet oil. J. Biol. Phys. Chem. 2013, 13, 69-72. [CrossRef]

8. Prajapati, A.; Bechtel, J.; Ganesan, S. Condition based maintenance: A survey. J. Qual. Maint. Eng. 2012, 18, 384-400. [CrossRef]

9. Zhmud, B.; Pasalskiy, B. Nanomaterials in Lubricants: An Industrial Perspective on Current Research. Lubricants 2013, 1, 95-101. [CrossRef]

10. Dai, W.; Kheireddin, B.; Gao, H.; Liang, H. Roles of nanoparticles in oil lubrication. Tribol. Int. 2016, 102, 88-98. [CrossRef]

11. Liu, Z.; Leininger, D.; Koolivand, A.; Smirnov, A.I.; Shenderova, O.; Brenner, D.W.; Krim, J. Tribological properties of nanodiamonds in aqueous suspensions: Effect of the surface charge. RSC Adv. 2015, 5, 78933-78940. [CrossRef]

12. Ivanov, M.G.; Ivanov, D.M.; Pavlyshko, S.V.; Petrov, I.; Vargas, A.; McGuire, G.; Shenderova, O. Nanodiamond-Based Nanolubricants. Fuller. Nanotubes Carbon Nanostruct. 2012, 20, 606-610. [CrossRef]

13. Raina, A.; Anand, A. Tribological investigation of diamond nanoparticles for steel/steel contacts in boundary lubrication regime. Appl. Nanosci. 2017, 7, 371-388. [CrossRef]

14. Tong, Y.; Liu, R.; Zhang, T. The effect of a detonation nanodiamond coating on the thermal decomposition properties of RDX explosives. Phys. Chem. Chem. Phys. 2014, 16, 17648-17657. [CrossRef] [PubMed]

15. Osei-Agyemang, E.; Berkebile, S.; Martini, A. Decomposition Mechanisms of Anti-wear Lubricant Additive Tricresyl Phosphate on Iron Surfaces Using DFT and Atomistic Thermodynamic Studies. Tribol. Lett. 2018, 66, 48. [CrossRef] 
16. Loehlé, S.; Righi, M. Ab Initio Molecular Dynamics Simulation of Tribochemical Reactions Involving Phosphorus Additives at Sliding Iron Interfaces. Lubricants 2018, 6, 31. [CrossRef]

17. De Barros-Bouchet, M.I.; Righi, M.C.; Philippon, D.; Mambingo-Doumbe, S.; Le-Mogne, T.; Martin, J.M.; Bouffet, A. Tribochemistry of phosphorus additives: Experiments and first-principles calculations. RSC Adv. 2015, 5, 49270-49279. [CrossRef]

18. Bancroft, G.M.; Kasrai, M.; Fuller, M.; Yin, Z.; Fyfe, K.; Tan, K.H. Mechanisms of tribochemical film formation: Stability of tribo- and thermally-generated ZDDP films. Tribol. Lett. 1997, 3, 47-51. [CrossRef]

19. Acharya, B.; Pardue, T.N.; Avva, K.S.; Krim, J. In Situ, real time studies of thermal reaction film formation temperatures for iron and $304 S S$ surfaces immersed in 5\% tricresyl phosphate in base oil. Tribol. Int. 2018, 126, 106-115. [CrossRef]

20. Barrat, J.-L.; Bocquet, L. Large Slip Effect at a Nonwetting Fluid-Solid Interface. Phys. Rev. Lett. 1999, 82, 4671-4674. [CrossRef]

21. Acharya, B.; Sidheswaran, M.A.; Yungk, R.; Krim, J. Quartz crystal microbalance apparatus for study of viscous liquids at high temperatures. Rev. Sci. Instrum. 2017, 88, 025112. [CrossRef] [PubMed]

22. Stanford Research System. QCM 100 Quartz Crystal Microbalance Analog Controller-QCM 25 Crystal Oscillator; Stanford Research Systems, Inc.: Sunnyvale, CA, USA, 2002.

23. Dusen, M.S. Van Platinum-resistance thermometry at low temperatures 1. J. Am. Chem. Soc. 1925, 47, 326-332. [CrossRef]

24. Krim, J.; Heyvaert, I.; Van Haesendonck, C.; Bruynseraede, Y. Scanning tunneling microscopy observation of self-affine fractal roughness in ion-bombarded film surfaces. Phys. Rev. Lett. 1993, 70, 57-60. [CrossRef] [PubMed]

25. Yuan, Y.; Lee, T.R. Contact Angle and Wetting Properties. In Surface Science Techniques; Bracco, G., Holst, B., Eds.; Springer: Berlin/Heidelberg, Germany, 2013; pp. 3-34.

26. Wang, L.; Wood, R.J.K. The influence of contact conditions on surface reaction layers formed between steel surfaces lubricated by an aviation oil. Tribol. Int. 2007, 40, 1655-1666. [CrossRef]

27. Curtis, C.K.; Marek, A.; Smirnov, A.I.; Krim, J. A comparative study of the nanoscale and macroscale tribological attributes of alumina and stainless steel surfaces immersed in aqueous suspensions of positively or negatively charged nanodiamonds. Beilstein J. Nanotechnol. 2017, 8, 2045-2059. [CrossRef] [PubMed]

28. Acharya, B.; Chestnut, M.; Marek, A.; Smirnov, A.I.; Krim, J. A Combined QCM and AFM Study Exploring the Nanoscale Lubrication Mechanism of Silica Nanoparticles in Aqueous Suspension. Tribol. Lett. 2017, 65, 115. [CrossRef]

29. Johannsmann, D. Viscoelastic, mechanical, and dielectric measurements on complex samples with the quartz crystal microbalance. Phys. Chem. Chem. Phys. 2008, 10, 4516-4534. [CrossRef] [PubMed]

30. Wang, J.; Pu, J.; Zhang, G.; Wang, L. Interface Architecture for Superthick Carbon-Based Films toward Low Internal Stress and Ultrahigh Load-Bearing Capacity. ACS Appl. Mater. Interfaces 2013, 5, 5015-5024. [CrossRef] [PubMed]

31. Wojciechowski, L.; Kubiak, K.J.; Mathia, T.G. Roughness and wettability of surfaces in boundary lubricated scuffing wear. Tribol. Int. 2016, 93, 593-601. [CrossRef]

32. Stepina, V.; Vesely, V. Lubricants and Special Fluids; Additives; Tribology Series; Elsevier: New York, NY, USA, 1992; Volume 23, pp. 255-407. [CrossRef]

33. Lancaster, J.K. A review of the influence of environmental humidity and water on friction, lubrication and wear. Tribol. Int. 1990, 23, 371-389. [CrossRef]

34. Abdelmaksoud, M.; Lee, S.M.; Padgett, C.W.; Irving, D.L.; Brenner, D.W.; Krim, J. STM, QCM, and the Windshield Wiper Effect: A Joint Theoretical-Experimental Study of Adsorbate Mobility and Lubrication at High Sliding Rates. Langmuir 2006, 22, 9606-9609. [CrossRef] [PubMed]

35. Zhang, J.; Spikes, H. On the Mechanism of ZDDP Antiwear Film Formation. Tribol. Lett. 2016, 63, 24. [CrossRef]

(c) 2018 by the authors. Licensee MDPI, Basel, Switzerland. This article is an open access article distributed under the terms and conditions of the Creative Commons Attribution (CC BY) license (http:/ / creativecommons.org/licenses/by/4.0/). 